\title{
Transmittance Photoplethysmography for Pulse Oximetry with Near Infrared Laser Diodes
}

\author{
S. M. López (TME), M. L. Dotor ${ }^{1}$, J. P. Silveira ${ }^{1}$, R. Giannetti ${ }^{2}$, D. Golmayo ${ }^{3}$, \\ P. Martín ${ }^{4}$, F. Miguel-Tobal ${ }^{4}$, A. Bilbao ${ }^{4}$, J.L. Álvarez-Sala ${ }^{5}$, L. Herrera ${ }^{6}$
}

\begin{abstract}
Photoplethysmography and pulse oximetry are widely used techniques for noninvasive monitoring of heart rate and peripheral oxygen saturation. In recent years we have been working on the application of near infrared range wavelengths to replace those of the classical pulse oximeters. The development of laser diodes based sensors, processing algorithms, a calibration procedure, and the comparison with a commercial pulse oximeter, have been followed by a set of experimental studies. The results presented here demonstrate the feasibility of transmittance photoplethysmography for pulse oximetry in both peripheral and non-peripheral beds under different conditions.
\end{abstract}

Index Terms-- laser diodes, optical sensor, photoplethysmography, pulse oximetry.

\section{INTRODUCTION}

$\mathrm{P}$ ULSE oximeters are used to provide non-invasive monitoring of arterial blood hemoglobin oxygen saturation $\left(\mathrm{So}_{2}\right)[1,2]$. This technique is based on time variable optical attenuation by a vascular bed due to cardiac pumping action (photoplethysmography) and the differential optical absorption of the oxy- $\left(\mathrm{HbO}_{2}\right)$ and deoxyhemoglobin $(\mathrm{RHb})$ at two specific wavelengths [3-5]. This approach assumes that the time dependent photoplethysmographic signal (PPG) is caused solely by changes in the arterial blood volume associated with the cardiac cycle and that no other hemoglobin derivatives different from $\mathrm{HbO}_{2}$ or $\mathrm{RHb}$ are present. The classical pulse oximeters [1-2] use light emitting diodes (LEDs) as sources, with emissions in two regions of the optical spectrum: in the red (e.g. $660 \mathrm{~nm})$ and in the infrared $(880-940 \mathrm{~nm})$. The pulse oximetry saturation can be obtained both from transmitted and reflected signals using sensors with the appropriate configuration.

Pulse oximetry is a well-established technique for monitoring peripheral oxygen saturation. Nevertheless, several publications have been devoted to its improvement and new applications. Among these is possible to find refined processing algorithms $[8,9]$ and the development of new sensors $[10,11]$. By contrast, the wavelengths used in pulse oximetry have not varied for decades and only in recent years some studies have focused on this issue. New wavelengths have been proposed [12-15], mainly to apply reflectance pulse oximetry to fetal monitoring more effectively $[14,15]$. The interference of hemoglobin derivatives such as carboxyhemoglobin ( $\mathrm{HbCO}$ ) has also been under analysis [16]. Some studies have used photoplethysmography and pulse oximetry in non-peripheral vascular beds, as esophagus $[17,18]$ and internal organs [19].

Jöbsis [20] first showed that near infrared (NIR) radiation penetrates excellently through biological tissues. After that several authors have applied NIR techniques to tissue oximetry [21]. One particular feature of the hemoglobin derivatives optical characteristics above $750 \mathrm{~nm}$ is the small absorption of the carboxyhemoglobin [22], being an expected advantage the minimal $\mathrm{HbCO}$ interference on pulse oximetry readings. Besides this, the measurement in the NIR band could facilitate monitoring pulsating vascular beds other than a fingertip or an infant foot under a normal situation. Thus, we have been working on the application of a NIR range wavelength closer to $760 \mathrm{~nm}$ to replace the "red wavelength" of the classical pulse oximeters. We proposed a transmittance optical sensor based on two NIR laser diodes and a whole measure system [23, 24]. Subsequently, we reported the development of processing algorithms [25, 26], optical sensor improvements, calibration procedure modifications and comparison with a commercial pulse oximeter [27-29]. It has been followed by sensor adaptation and application of the system to the monitoring of peripheral and non-peripheral vascular beds [30-32]. In this work we present the study of athletes over stress tests and pig intra-peritoneal organs by transmittance photoplethysmography for pulse

\footnotetext{
${ }^{1}$ Instituto de Microelectrónica de Madrid, CNM, CSIC

${ }^{2}$ DEA, Universidad Pontificia Comillas de Madrid

${ }^{3}$ Instituto de Ciencia de Materiales de Madrid, CNM, CSIC

${ }^{4}$ Escuela Profesional de Medicina de la Educación Física y el Deporte, Universidad Complutense de Madrid

${ }^{5}$ Hospital Clínico San Carlos, Facultad de Medicina, Universidad Complutense de Madrid

${ }^{6}$ Hospital Universitario Marqués de Valdecilla, Facultad de Medicina, Universidad de Cantabria
} 
oximetry with the proposed system.

\section{METHODS}

\section{A. Pulse Oximetry Principles}

Oxygen saturation is obtained by analyzing the pulsating component peak-to-peak value $\left(E_{A C}\right)$ of the PPG related to the corresponding constant component $\left(E_{D C}\right)$ of the PPG, at two specific wavelengths. Some theoretical equations assume the validity of Beer-Lambert-Bouguer's law for deriving the relationship between oxygen saturation $\left(\mathrm{So}_{2}\right)$ and the optical properties of a pulsating vascular bed as [3-7]:

$$
S o_{2}=\frac{\varepsilon_{R H b}^{\lambda_{1}}-\varepsilon_{R H b}^{\lambda_{2}} \cdot q}{\varepsilon_{R H b}^{\lambda_{1}}-\varepsilon_{H b O_{2}}^{\lambda_{1}}-\left(\varepsilon_{R H b}^{\lambda_{2}}-\varepsilon_{H b O_{2}}^{\lambda_{2}}\right) \cdot q}
$$

where $\varepsilon_{\mathrm{RHb}}^{\lambda_{1}}, \varepsilon_{\mathrm{HbO}_{2}}^{\lambda_{1}}, \varepsilon_{\mathrm{RHb}}^{\lambda_{2}}, \varepsilon_{\mathrm{HbO}}^{\lambda_{2}}$ are the absorptivities or specific absorption coefficients of RHb and

$\mathrm{HbO}_{2}$ at wavelengths $\lambda_{1}$ and $\lambda_{2}$ respectively, and $q$ is the ratio of the signals $E_{A C}$ and $E_{D C}$ for these wavelengths [3-5], which could be expressed as:

$$
q=\frac{\log \left(1-\frac{E_{A C}^{\lambda 1}}{E_{D C}^{\lambda 1}}\right)}{\log \left(1-\frac{E_{A C}^{\lambda 2}}{E_{D C}^{\lambda 2}}\right)} \approx \frac{\frac{E_{A C}^{\lambda 1}}{E_{D C}^{\lambda 1}}}{\frac{E_{A C}^{\lambda 2}}{E_{D C}^{\lambda 2}}}
$$

The attenuation of the optical radiation by a pulsate vascular bed is due to blood $\mathrm{RHb}$ and $\mathrm{HbO}_{2}$ absorption, but also to multiple scattering in the red blood cells and tissue structures [6,7]. Thus, in reality, the Eq. (1) is an approximation and from a practical point of view, a typical relationship used for calibrating pulse oximeters has the form [7]:

$$
\mathrm{So}_{2}=\frac{K_{1}-K_{2} \bullet q}{K_{3}-K_{4} \bullet q}
$$

where $K_{1}, K_{2}, K_{3}$ and $K_{4}$ are coefficients derived from the pulse oximeter calibration procedure that are related to the specific absorption coefficients of $\mathrm{HbO}_{2}$ and $\mathrm{RHb}$.

\section{B. Measure System}

The whole measure system comprises the optical sensor, sensor electronics, and elaboration unit (Fig. 1). The configuration of the developed optical sensors (Fig. 2 (a)) corresponds to the transmission mode, being the emitters (LD) and the photo-detectors (PD) situated in opposite sides with respect to the vascular bed analyzed. Two laser diodes with peak wavelengths close to $750 \mathrm{~nm}$ and $850 \mathrm{~nm}$ are used as optical emitters, which match the optical absorption characteristics of $\mathrm{HbO}_{2}$ and $\mathrm{RHb}$ [23]. In the early version of the optical sensor the LDs were mounted in their own original metal substrate (separation: 7 $\mathrm{mm}$ ) $[23,24]$. In the second prototype [27-29], to reduce the mismatch in the volumes probed by each wavelength and to minimize the movement artifacts, the two LD chips are mounted on a single metal heat sink with a separation distance between their central parts of $0.7 \mathrm{~mm}$. Both chips are situated so that their emission beam larger divergence axes are parallel to the sensor central longitudinal axis and three BPW34 pi-n silicon photodiodes are connected in parallel. In the PD backside is the first amplifier stage, which converts the photocurrent into a proportional voltage. A third prototype has been developed for fingertip transmittance measurements in athletes over stress tests [31]. In this sensor, both LDs are situated with their emission beam larger divergence axes on the sensor central axis. 


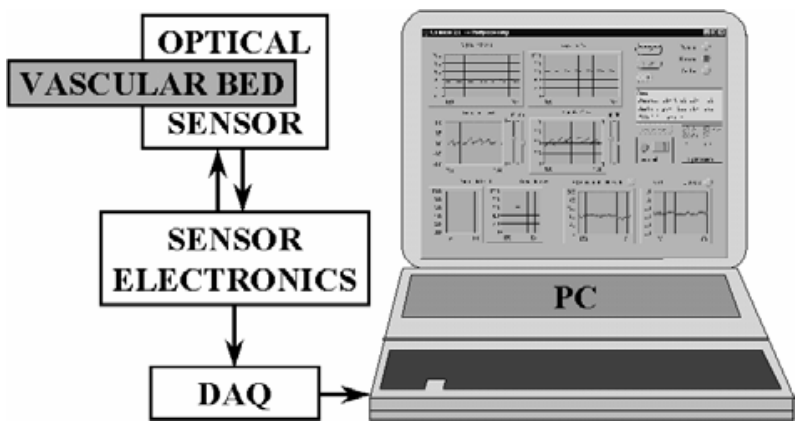

Fig. 1. Scheme of the whole measure system for photoplethysmography and pulse oximetry. The analyzed vascular bed is placed between both sides of the transmittance optical sensor. The laser-diodes driver, amplification stages, timing and sample-and-hold circuits, constitute the sensor electronics. The data acquisition board (DAQ) and the programs installed on a personal computer (PC) form the signal processing block.

The laser-diodes driver, amplification stages, timing and sample and hold (S\&H) circuits, constitute the sensor electronics (Fig. 1). The laser diodes driver sequentially activates each LD during $5 \mu$ s at a repetition rate of $1 \mathrm{kHz}$. The outputs of the S\&H are fed into the analogue inputs of a 12-bit data acquisition board (DAQ). The DAQ and the processing programs installed on a handheld personal computer (PC, Fig. 1) constitute the elaboration unit. The timing board of the sensor electronics also generates the trigger and conversion signals for the DAQ. The S\&H output signals are analogically pre-filtered by the DAQ with a simple anti-aliasing RC low pass filter at $300 \mathrm{~Hz}$ and after that they are digitized at $1 \mathrm{kSa} / \mathrm{s}$ for every channel. The next stages of the signal processing are carried out digitally, either in real time or offline (post-processing), after a ten-sample averaging of each wavelength PPG signal.

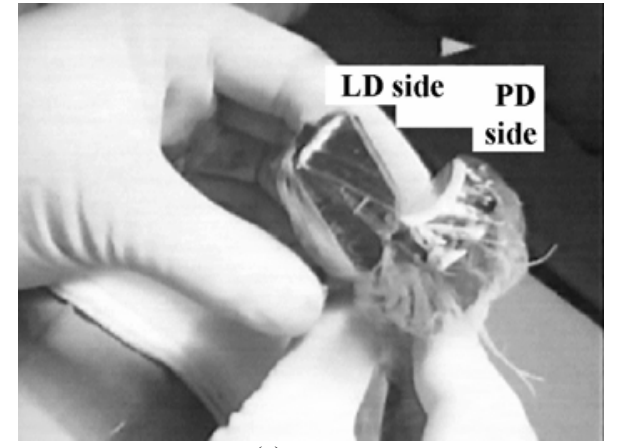

(a)

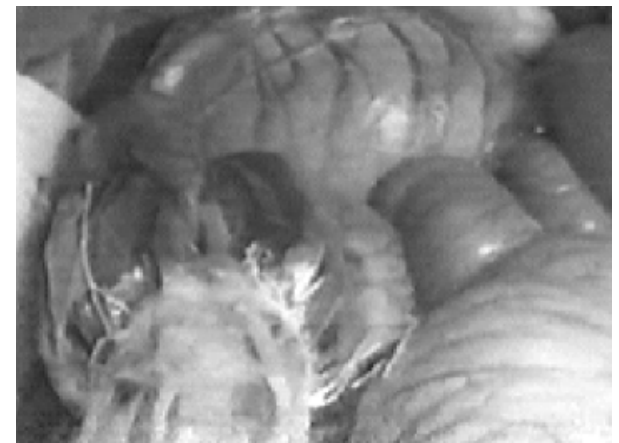

(b)

Fig. 2. The near infrared laser diode-based transmittance optical sensor. View of the laser diode (LD) and the photodiode (PD) sides of the optical sensor (a). The second prototype of the LD transmittance sensor is inserted in a sterilized protection plastic and fixed to pig intra-abdominal viscera (b).

According to Eq. (2), each wavelength PPG signal has to be decomposed into its variable or pulsating component $(A C)$ and the constant or non-pulsating component $(D C)$. By using a real time implemented algorithm [25, 26], for a given time interval, the constant component ( $E_{D C}$, Eq. 2$)$ and the pulsating component peak-to-peak value ( $E_{A C}$, Eq. 2) are obtained for each wavelength by a low pass Bessel filtering, and a band pass Bessel filtering followed by a nonlinear one, respectively. The derived quotient of both $E_{A C} / E_{D C}$ ratios (q, Eq. 2) is related to $\mathrm{So}_{2}$.

A medically supervised study was conducted in the Pneumology Department of the Hospital Clínico San Carlos of Madrid (Spain) to calibrate the proposed system with the second cited sensor as a pulse oximeter [27-29]. After approval by the Ethics Committee of the Hospital, 41 patients with respiratory failure who required specific arterial blood determinations as a part of their conventional medical attention were studied by fingertip transmittance with our system and a commercial pulse oximeter to cover a wide range of $\mathrm{So}_{2}$ values. Thus, a calibration function, similar to Eq. (3), was derived with a significant quantity of experimental points, ranging the in vitro arterial $\mathrm{So}_{2}$ from 60 to $95 \%$ and using the above-described algorithm $[25,26]$. This calibration curve is specific for the sensor emitters and configuration, as well as for the signal processing procedure. The standard deviation of the differences in the oxygen saturation values obtained with the NIR laser diodes based system and the arterial blood analysis was $3.1 \%[27,29]$. 


\section{Monitoring of Athletes over Stress Tests}

After the adaptation of the developed near-infrared laser diodes based system, an extended measurement campaign has been carried out [30] in a group of athletes in order to gain into the knowledge of the physiological mechanisms during the effort. There, it is a great interest to monitor the blood oxygen saturation during exercise in order to improve the sport training. The main problem is the apparition of many movement artifacts. We optimize the sensor attachment in order to reduce the random artifacts, obtaining PPG signals with only two variable components: the cardiac pulse and the rhythmic movement of the body during running. To study these subjects we monitored the blood oxygen saturation and the heart rate using a commercial pulse oximeter and our system. In addition to that, electrocardiography (ECG) signal, blood pressure, and breathing exhaled gas analysis were also monitored. The measurements were performed at the Laboratory of Physiology of the Professional School of Sport Medicine (Complutense University, Madrid). Each of the oximeter sensors was attached to an athlete's finger, and then a latex glove was used to keep in place the probe. Once prepared after connecting the measurement electrodes and tubes, a complete maximal exercise test by treadmill ergometer and cycling ergometer, was performed on each of the subjects.

\section{Photoplethysmography in Intra-Peritoneal Organs}

A preliminary animal study was conducted in the Laboratory of Experimental Surgery at the Faculty of Medicine of the University of Cantabria (Spain) [31,32]. The study was performed in intra-peritoneal organs of a Large-White pig with a weight of $16 \mathrm{~kg}$, which was obtained and manipulated according to the Animal Welfare Act ethical statements for research animals [34]. The pig was pre-medicated with 15 $\mathrm{mg}$ per kg Ketamine and $0.1 \mathrm{mg}$ atropine, intramuscularly. Induction of general anesthesia was performed with $10 \mathrm{mg}$ per $\mathrm{kg}$ sodium pentothal, which were injected intravenously. It was followed by an endotracheal intubation. Anesthesia was maintained after intubation by $\mathrm{NO}_{2}$ and $\mathrm{O}_{2}$ breath at 61 per minute and $0.075 \mathrm{mg}$ per $\mathrm{kg}$ fentanyl plus $2 \mathrm{mg}$ per 20-30 minutes pancuronium bromide administered intravenously. A laparotomy under conventional asepsis conditions permitted to reach the intraabdominal viscera.

The transmittance sensor was inserted in a sterilized protection plastic and then fixed to specific intraperitoneal organs, as shown in figure 1(b). Prior to register the PPG, each laser diode injection current was adjusted to avoid either detector saturation or low signal levels. The injection current was higher than the threshold current $\left(\mathrm{I}_{\mathrm{th}}\right)$ with injection values between $1.5 \cdot \mathrm{I}_{\mathrm{th}}$ and $2.5 \cdot \mathrm{I}_{\mathrm{th}}$. Two-wavelength PPG signals (LD - $850 \mathrm{~nm}$ and LD - $750 \mathrm{~nm}$ ) were recorded in the mesocolon, mesentery root, gastric wall and aorta. ECG and arterial pressure were continuously monitored, being their values 150 beats per minute and $120 / 90$ respectively over the whole experiment. At the conclusion of the experiment, the pig was sacrificed with $\mathrm{KCl}$ intravenously and ventricular fibrillation.

\section{RESULTS}

\section{A. Monitoring of Athletes over Stress Tests}

A method that comprises the non-linear filtering used in the developed oximetry system $[25,26]$ with an off-line frequency domain analysis has been designed in order to obtain a valid value of the oxygen related parameter (q, Eq. 2) from the recorded photoplethysmographic signals [30]. The heart rates (beats per minute, bpm) obtained with the IMM laser diodes based system (IMM NIR-LD), the commercial LED based pulse oximeter and ECG are shown in figure 3. The LED pulse oximeter either overestimates or underestimates the heart rate in comparison with the ECG record. By contrast, the IMM NIR-LD system follows the ECG behavior pattern.

\section{B. Photoplethysmography in Intra-Peritoneal Organs}

In figure 4 are presented raw PPG recorded with $\mathrm{LD}-750 \mathrm{~nm}$ and $\mathrm{LD}-850 \mathrm{~nm}$ in $10 \mathrm{~s}$ intervals in the mesocolon (4a) and the aorta (4b). The aorta signals have large peak-to-peak pulsating components with few spikes in comparison to those signals from mesocolon, mesentery root and gastric wall. Additional oscillations, which seem to be related to the respiratory rate, are clearly observed in the aorta PPG signals over the whole time interval. The $E_{A C}$ to $E_{D C}$ ratios are around $30 \%$ for aorta and below $15 \%$ for the other three organs. 


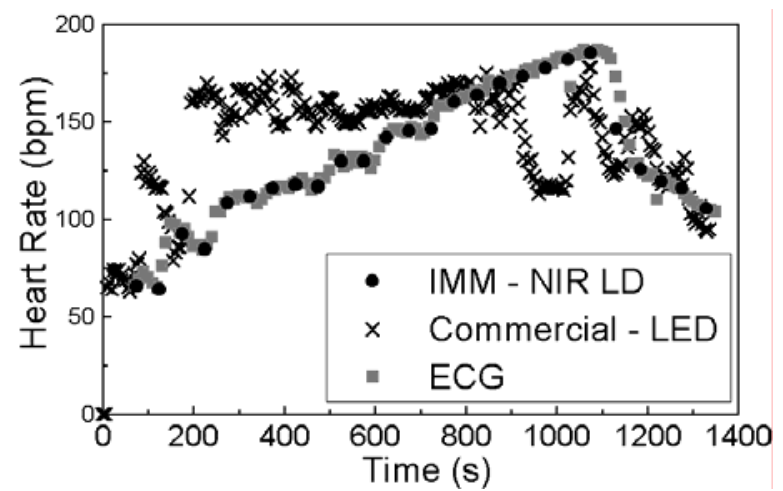

Fig. 3. The heart rates (beats per minute, bpm) obtained with the laser diode-based system (IMM - NIR LD), a commercial LED based pulse oximeter and electrocardiography (ECG). The IMM - NIR LD system follows the ECG behavior pattern, while the LED pulse oximeter either overestimates or underestimates the heart rate in comparison with the ECG record.

A Fast Fourier Transformation (FFT) analysis of the raw PPG signals with a Hanning window has shown, for both laser diodes LD - $750 \mathrm{~nm}$ and LD - $850 \mathrm{~nm}$ (Fig. 5a), a clear peak frequency around 2.5 $\mathrm{Hz}$ in all of the studied organs (aorta, mesocolon, mesentery root and gastric wall). Despite of the differences in the raw PPG, this peak frequency is in coincidence with the $150 \mathrm{bpm}$ of the ECG record. Figure 5b shows the FFT analysis in $10 \mathrm{~s}$ intervals every $5 \mathrm{~s}$ over $60 \mathrm{~s}$ of PPG signals recorded in the gastric wall using the laser diode LD - $750 \mathrm{~nm}$. The peak frequency around $2.5 \mathrm{~Hz}$ is stable over the whole time interval and a similar response is observed for the laser diode LD - $850 \mathrm{~nm}$ and in all of the organs.

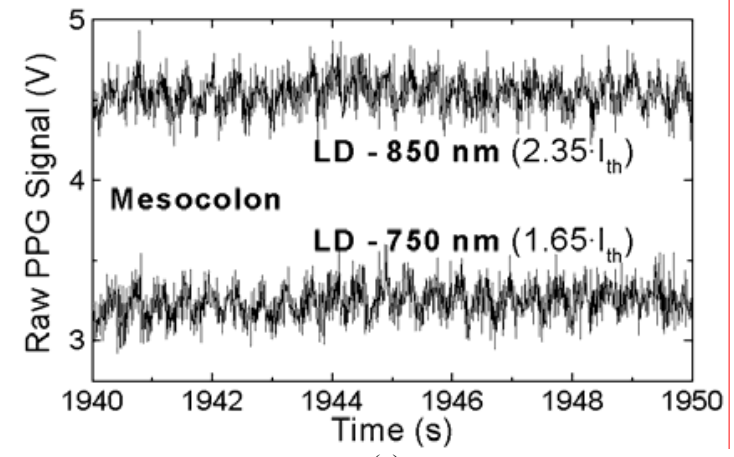

(a)

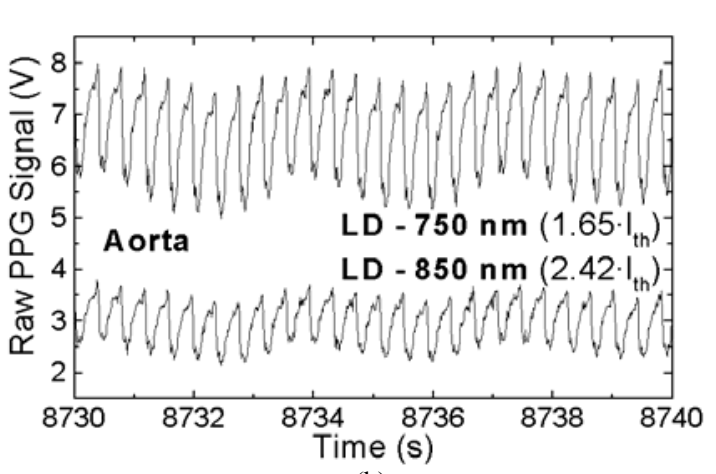

(b)

Fig. 4. Raw transmittance photoplethysmographic (PPG) signals recorded over 10 seconds' intervals in the pig mesocolon (a) and aorta (b) using the laser diodes $\mathrm{LD}-750 \mathrm{~nm}$ and $\mathrm{LD}-850 \mathrm{~nm}$. The injection current of each $\mathrm{LD}$ was adjusted to a value between 1.5 and 2.5 times the threshold current $\left(\mathrm{I}_{\mathrm{th}}\right)$ to avoid either detector saturation or low PPG signal levels.

The measured photoplethysmographic signals have sufficient amplitudes at both wavelengths from various intra-peritoneal organs to make pulse oximetry feasible. The preliminary derived heart rates are in agreement with the reference value (ECG). Nevertheless, further improvements and studies are necessary in order to develop optimized sensors and processing algorithms for real time monitoring.

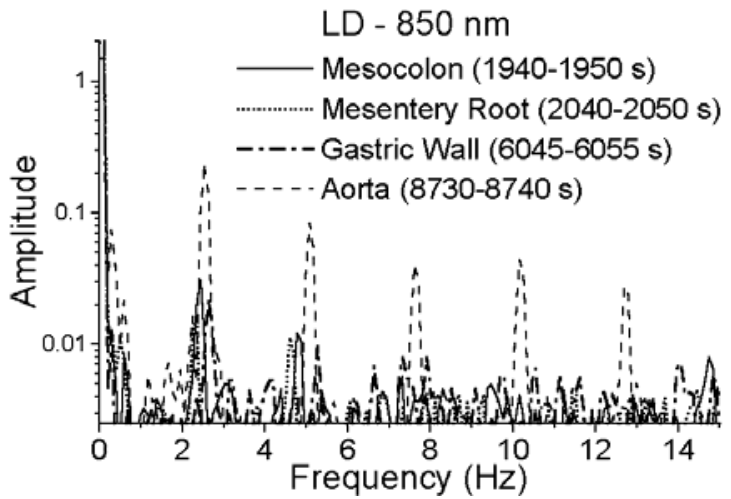

(a)

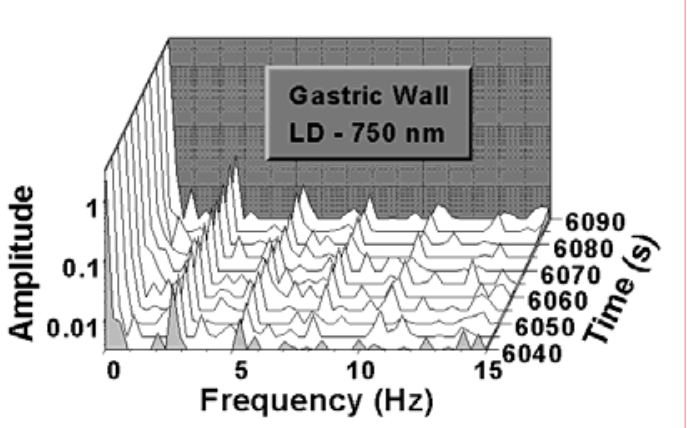

(b)

Fig. 5. FFT analysis of the raw transmittance photoplethysmographic signals. The analysis of signals form the laser diode (LD) $850 \mathrm{~nm}$ recorded over $10 \mathrm{~s}$ intervals $(\mathrm{a})$ in the pig mesocolon $(-)$, mesentery root $(\cdots)$, gastric wall (--) and aorta (---). FFT results for PPG signals in $10 \mathrm{~s}$ intervals every $5 \mathrm{~s}$ over $60 \mathrm{~s}$ recorded using the laser diode $750 \mathrm{~nm}$ in the gastric wall (b). 


\section{CONClusions}

The results presented here demonstrate the feasibility of transmittance photoplethysmography with laser diodes emitting at specific NIR wavelengths for pulse oximetry in a variety of situations. The flexibility of the developed system let us to use sensors adapted for different kind of measurements in several places, organs and situations. For example: to measure the heart rate and absolute oxygen saturation value in a fingertip (peripheral pulsating bed) under basal conditions or over a stress test, and to record the variations in intra-peritoneal organs (non-peripheral pulsating bed). Once the raw signals are acquired, the processing by software corresponds to the type of measurement. It is possible to convert the $q$ values obtained after the processing of the photoplethysmographic signals measured at both NIR wavelengths in the corresponding levels of oxygen saturation by taking into account the established calibration.

\section{ACKNOWLEDGMENTS}

This work has been partially supported by the Spanish Commission for Science and Technology (CICYT) project TIC98-1025, the grants 04/EPB10/02 and 02/EPB10/03 from the Education, Culture and Sport Ministry of Spain, the University of Las Palmas de Gran Canaria project UNI 2002/15, the University of Cantabria, and Asociación Cántabra de Cirugía (Santander, Spain). Spanish Programa Ramón y Cajal supports SMLS. The authors wish to thank Dr. David Shea, technical translation lecturer at the University of Las Palmas de Gran Canaria for his editorial assistance in preparing the final manuscript.

\section{REFERENCES}

[1] L. G. Lindberg, C. Lennmarken and M. Vegfors, "Pulse oximetry - clinical implications and recent technical developments". Acta Anesthesiol. Scand., vol. 39, pp. 279-287, 1995.

[2] J. T. B. Moyle, "Uses and abuses of pulse oximetry". Arch. Dis. Child., vol. 74, pp. 77-80, 1996.

[3] T. Aoyagi, M. Kishi, K. Yamaguchi and S. Watanabe, "Improvement of an earpiece oximeter". In: Abstracts of the Japanese Society of Medical Electronics and Biological Engineering, pp. 90-91, 1974.

[4] I. Yoshiya, Y. Shimada and K. Tanaka, "Spectrophotometric monitoring of arterial oxygen saturation in the fingertip". Med. Biol. Eng. Comp., vol. 18, pp. 27-32, 1980.

[5] I. Yoshiya and Y. Shimada, "Non-invasive spectrophotometric estimation of arterial oxygen saturation". In: Noninvasive physiological measurements, Academic, New York, pp. 251-286, 1983.

[6] J. M. Schmitt, "Simple photon diffusion analysis of the effects of multiple scattering on pulse oximetry". IEEE Trans. Biomed. Eng., vol. 38, pp. 1194-1203, 1991.

[7] Y. Mendelson and J. C. Kent, "Variations in optical absorption spectra of adult and fetal hemoglobin and its effects on pulse oximetry”. IEEE Trans. Biomed. Eng., vol. 36, pp. 844-848, 1989.

[8] F. M. Coetzee and Z. Elghazzawi, "Noise-resistant pulse oximetry using a synthetic reference signal". IEEE Trans. Biomed. Eng., vol. 47, pp. 1018-1026, 2000.

[9] M. J. Hayes and P. R. Smith, "A new method for pulse oximetry possessing inherent insensitivity to artifact". IEEE Trans. Biomed. Eng., vol. 48, pp. 452-461, 2001.

[10] M. Nogawa, T. Kaiwa and S. Takatani, "A novel hybrid reflectance pulse oximeter sensor with improved linearity and general applicability to various portions of the body". Proc. 20th Ann. Int. Conf. IEEE/EMBS, vol. 4, pp. 1858-1861, 1998.

[11] S. Rhee, B. H. Yang, and H. H. Asada, "Artifact-resistant power-efficient design of finger-ring plethysmographic sensors". IEEE Trans. Biomed. Eng., vol. 48, pp. 795-805, 2001.

[12] I. Fine, A. Sternberg, Y. Katz, L. Goldinov and B. Rapoport, "Sensor, method and device for optical blood oximetry". WO 9641566 A2 961227, 1996.

[13] P. D. Mannheimer, J. R. Casciani, M. E. Fein and S. L. Nierlich, "Wavelength selection for low saturation pulse oximetry". IEEE Trans. Biomed. Eng., vol. 44, pp. 148-158, 1997.

[14] R. M. Lewinsky and I. Fine, "Antepartum fetal pulse oximetry”. Eur. J. Obstet. Gynecol. Reprod. Biol., vol. 72 Suppl 1, S81-S85, 1997.

[15] P. D. Mannheimer, M. E. Fein and J. R. Casciani, "Physio-optical considerations in the design of fetal pulse oximetry". Eur. J. Obstet. Gynecol. Reprod. Biol., vol. 72, Suppl 1, S9-S19, 1997.

[16] N. B. Hampson, "Pulse oximetry in severe carbon monoxide poisoning". Chest, vol. 114, pp. 1036-1041, 1998.

[17] M. N. Vincenzi, H. Gombotz, H. Krenn, C. Dorn and P. Rehark, "Transophageal versus surface pulse oximetry in intensive care unit patients". Crit. Care Med., vol. 28, pp. 2268-2270, 2000.

[18] P. A. Kyriacou, S. Powell, R. M. Langford and D. P. Jones, "Esophageal pulse oximetry utilizing reflectance photoplethysmography". IEEE Trans. Biomed. Eng., vol. 49, pp. 1360-1368, 2002.

[19] A. J. Crerar-Gilbert, P.A. Kyriacou, D. P. Jones, R. M. Langford, "Assessment of photoplethysmographic signals for the determination of splanchnic oxygen saturation in humans". Anaesthesia, vol. 57, pp. 442-445, 2002.

[20] F. F. Jöbsis, "Noninvasive, infrared monitoring of cerebral and myocardial oxygen sufficiency and circulatory parameters". Science, vol. 198, pp. 1264-1267, 1977.

[21] M. Ferrari, D. T. Delpy and D. A. Benaron, "Special section editorial: Near infrared spectroscopy and imaging of tissues". J. Biomed. Opt., vol. 1, pp. 361, 1996.

[22] W. G. Zijlstra, A. Buursma and W. P. Meeuwsen van der Roest, “Absorption spectra of human fetal and adult oxyhemoglobin, deoxyhemoglobin, carboxyhemoglobin and methemoglobin”. Clin. Chem. vol. 37, pp. 1633-1638, 1991. 
[23] S. M. López Silva, "Oximetría de pulso con diodos láser infrarrojos". Tesis Doctoral, Universidad Complutense de Madrid, 1997, http://www.iuma.ulpgc.es/users/slopez.

[24] S. M. López Silva, R. Giannetti, M. L. Dotor, J. R. Sendra, J. P. Silveira and F. Briones, "Application of NIR laser diodes to pulse oximetry". Proc. SPIE, vol. 3570, pp. 294-302, 1998.

[25] R. Giannetti, S. M. López Silva, M. L. Dotor, J. R. Sendra, J. P. Silveira and F. Briones, "An innovative signal processing algorithm for near infrared laser-based pulse oximeter". Proc. IMEKO TC-4, pp. 153-156, 1998.

[26] R. Giannetti, S. M. López Silva, M. L. Dotor, J. R. Sendra, J. P. Silveira, D. Golmayo and F. Briones, "Real time processing algorithm for a new NIR laser pulse oximeter". Proc. ESEM-99, pp. 217-218, 1999.

[27] J. P. Silveira, S. M. López Silva, M. L. Dotor, D. Golmayo, R. Giannetti, J. R. Sendra y J. L. Álvarez-Sala, "Oximetría de pulso basada en diodos láser infrarrojos". In: Libro de Actas de CASEIB 2000, pp. 13-16, 2000.

[28] S. M. López Silva, J. P. Silveira, J. R. Sendra, R. Giannetti, M. L. Dotor and D. Golmayo, "NIR transmittance pulse oximetry system with laser diodes". Proc. SPIE, vol. 4255, pp. 80-87, 2001.

[29] S. M. López Silva, M. L. Dotor and J. P. Silveira, "NIR transmittance pulse oximetry with laser diodes". J. Biomed. Opt., vol. 8, pp. 525-533, 2003.

[30] R. Giannetti, J. P. Silveira, M. L. Dotor, D. Golmayo, P. Martín, F. Miguel-Tobal, A. Bilbao, and S. M. López-Silva, "Oxygen saturation measurements in athletes attaining maximal exertion conditions". Proc. 21th IEEE/IMTC, vol. 1, pp. 740-744, 2004.

[31] S. M. López Silva, J. P. Silveira, L. Herrera y M. L. Dotor, "Fotopletismografía con diodos láser infrarrojos en órganos intraperitoniales del cerdo". Actas de la $7^{a}$ Reunión Nacional de Óptica, pp. 310-312, 2003.

[32] S. M. López-Silva, M. L. Dotor, J. P. Silveira, R. Giannetti, D. Golmayo, P. Martín, F. Miguel-Tobal, A. Bilbao, J. L. Álvarez-Sala and L. Herrera "Transmittance photoplethysmography and pulse oximetry with near infrared laser diodes", Proc. 21th IEEE/IMTC, vol. 1, pp. 718-723. 2004. 